
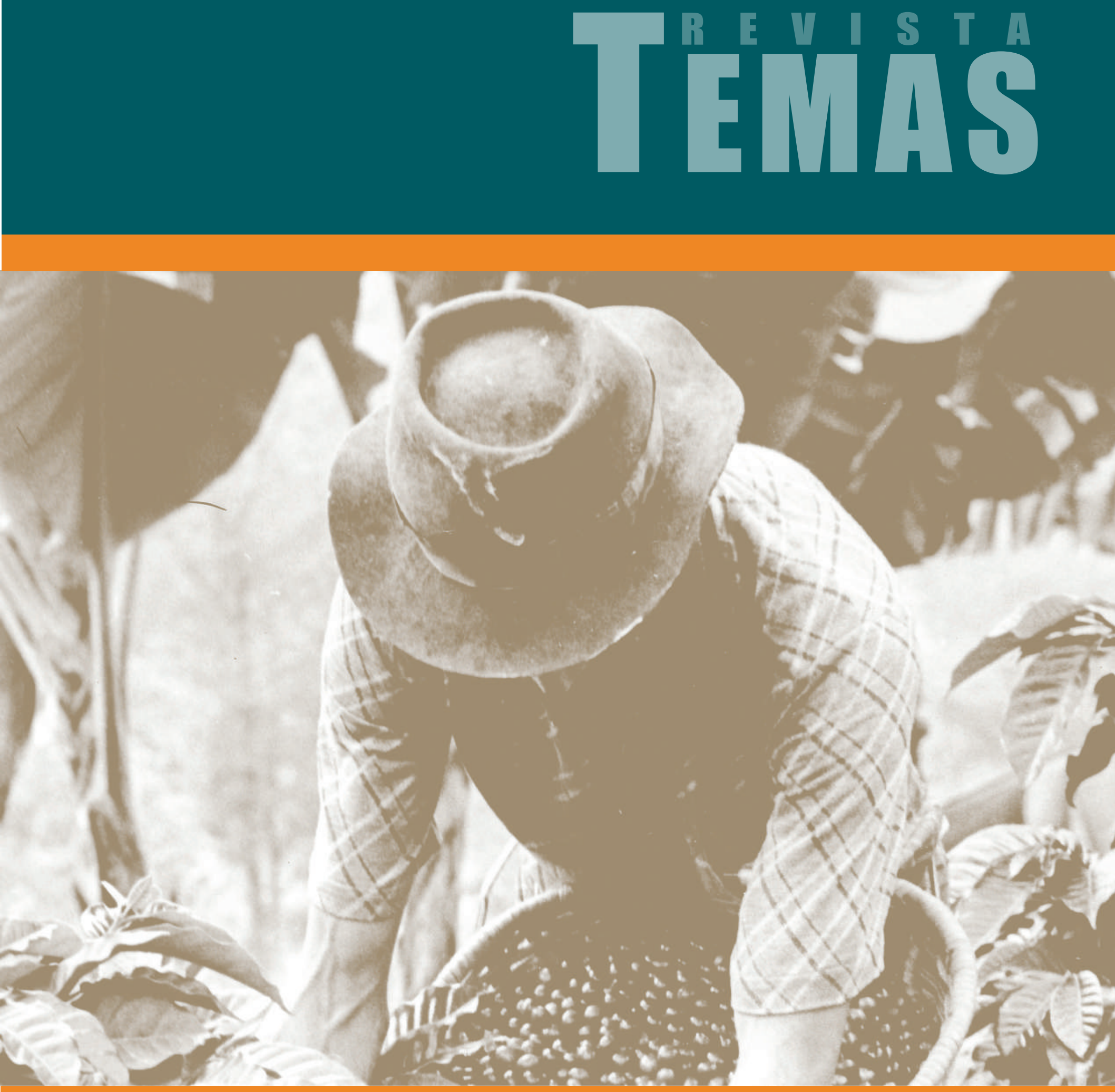



\section{EL FERROCARRIL DE CÚCUTA: LA GRANDEZA DE UNA ÉLITE LIBERAL 1}

(Recepción: Mayo 29 de 2013- Aceptación: Junio 26 de 2013)

Yesyd Fernando Pabón Serrano*

\section{Resumen}

El siguiente artículo analiza el proceso económico y empresarial que concluyó con la ejecución del proyecto vial en el Departamento del Norte de Santander, partiendo de la construcción de los primeros caminos que comunicaban la frontera colombo venezolana en el trayecto que conduce de la ciudad de Cúcuta al Puerto de Maracaibo en Venezuela, desde mediados de la década de 1860. De igual forma aborda las condiciones que más adelante posibilitaron la construcción de un ferrocarril sobre esa misma ruta del camino carretero, como lo fueron el crecimiento del cultivo y comercio del café, y el aumento de la importación de mercaderías desde el exterior para su comercialización en la región. En este sentido, la conformación de la Empresa del Ferrocarril de Cúcuta, su involucramiento en el desarrollo de la Guerra de los Mil Días y el proceso de desmonte de la Compañía son igualmente tratados para comprender la génesis de dicha empresa.

\section{Palabras clave}

Ferrocarril de Cúcuta, Compañía, Guerra Civil, Café, Frontera

\section{CÚCUTA RAILWAY: THE GREATNESS OF A LIBERAL ELITE}

\section{Alsstract}

The following article discusses the economic and business process which led to the implementation of the road project in the Department of Norte de Santander, based on the construction of the first roads that connected the Colombian-Venezuelan border in the path that leads to the city of Cucuta port of Maracaibo in Venezuela, from the mid1860s. Similarly addresses the conditions that later allowed the construction of a railroad on the same path wagon road, as were crop growth and coffee trade, and increasing the import of goods from abroad for marketing in region. In this sense, the formation of Cucuta Railway Company, their involvement in the development of the Thousand Days War and the process of clearing the Company are treated equally to understand the genesis of the company.

\section{Keywords}

Railway Cucuta Company, Civil War, Coffee, Frontier.

1 Artículo de Investigación: "Desde el camino a San Buenaventura hasta la Compañía del Ferrocarril: Historia del Ferrocarril de Cúcuta 1845 -1959", trabajo presentado para optar al título de Magíster en Historia de la Universidad Industrial de Santander 2011.

* Economista y Magister en Historia de la Universidad Industrial de Santander (UIS), Docente investigador Unidades Tecnológicas de Santander (UTS), Docente Auxiliar Facultad de Economía Universidad Autónoma de Bucaramanga (UNAB). Email: yefepase@hotmail.com 


\section{Introducción}

El siguiente artículo analiza el proceso mediante el cual se desarrolló el proyecto vial norte santandereano desde la construcción del camino al Puerto de San Buenaventura (actualmente Puerto Villamizar) a mediados de la década de 1860, hasta la posterior construcción del Ferrocarril de Cúcuta sobre la misma vía del camino. Inicialmente se aborda el origen del cultivo y comercialización del café en la región fronteriza que motivó el diseño y construcción del camino carretero y posteriormente del ferrocarril; luego se analiza el proceso que condujo al gobierno del Estado Soberano de Santander a adjudicar el privilegio para llevar a cabo las obras hacia el puerto fluvial; finalmente se analiza la evolución de la Compañía del Ferrocarril de Cúcuta y las causas que llevaron a la liquidación y desmantelamiento de la que fuera la empresa ferrocarrilera más importante de finales del Siglo XIX y principios del $X X$ en la región fronteriza y en Colombia.

En Colombia el desarrollo de las vías de comunicación y de los medios de transporte durante el siglo XIX se caracterizó por su atraso, ya que los caminos y los ríos navegables como el Magdalena eran la única alternativa para el desarrollo de la actividad comercial. Los proyectos viales más urgentes e idóneos para el país tropezaron con diversos obstáculos, el trazado y construcción de los primeros caminos y ferrocarriles y la construcción del Canal de Panamá dieron cuenta de ello. Como en gran parte de los países de América durante el siglo $\mathrm{XIX}$, Colombia tuvo una preferencia por la utilización de los ferrocarriles sobre los otros medios de comunicación, que beneficiaban directamente a algunos sectores comerciales nacionales y a empresas inglesas y norteamericanas que proveían a las compañías férreas las máquinas, herramientas y repuestos a cambio de cuantiosos empréstitos y el desarrollo de su industria pesada.
Cuando el actual Departamento del Norte de Santander formó parte del Estado Soberano, se inició en la región un proceso de expansión vial que involucró directamente a amplios sectores interesados en disfrutar las ventajas que la agricultura y el comercio ofrecían en la región ${ }^{2}$. Un producto en especial hizo posible la inserción de la economía regional en el ámbito nacional y externo; el café hizo su aparición por estas tierras y el crecimiento gradual de su cultivo y comercialización vitalizaron las relaciones económicas y humanas del departamento fronterizo, como ningún otro producto había logrado hacerlo en la época.

El Norte de Santander fue la primera región colombiana en incursionar en el cultivo y comercialización del café, desde aquí avanzó hacia las tierras del sur por la cordillera, donde lo acogieron Caldas y Antioquia. La ciudad de Cúcuta pionera en la exportación de café tomó ventaja en el manejo de los precios del mercado, aunque con algunas variaciones en las décadas de 1860 y 1870 logró aumentar el volumen de ventas. En el Norte del Estado Soberano se había empezado la producción de café desde 1820, aunque las primeras plantaciones se dedicaban al consumo doméstico; pero fue a partir de 1850 que las granjas experimentales se convirtieron en cultivos de café que produjeron un excedente suficientemente amplio para ser destinado a la exportación.

El origen del cultivo del café en Colombia estuvo matizado por la aparición de cultivos experimentales dispersos por diferentes regiones del territorio nacional; sin embargo el desarrollo del cultivo como lo pudieron evidenciar varios viajeros del siglo XIX, adquirió especial desarrollo en tierras santandereanas. Tal como lo

2 Un número considerable de comerciantes, dirigentes políticos, militares, empresarios y comerciantes de ambos lados de la región fronteriza colombo-venezolana fueron participes de los principales proyectos viales de la región. 
indica la tabla 1, para el año de 1874 la producción de café estaba concentrada principalmente en los territorios del actual Departamento de Norte de Santander y en menor proporción en Santander y Cundinamarca, era escaso o hasta desconocido el producto en otras regiones colombianas (Junguito, 1991).

Tabla 1. Producción de café por Departamentos en fechas seleccionadas entre 1874 y 1932 (miles de sacos de 60 kilos)

\begin{tabular}{|c|c|c|c|c|c|c|c|}
\hline AÑOS & 1874 & 1890 & 1892 & 1900 & 1913 & 1925 & 1932 \\
\hline & $B$ & 0 & $A$ & $\mathrm{O}$ & $B$ & $A$ & U-A \\
\hline Antioquia & 1.2 & 5 & 9.5 & 90 & 185 & 514 & 617 \\
\hline Boyacá & 0.6 & * & * & * & 3 & * & 23 \\
\hline Caldas & 1.3 & (a) & 2.7 & (a) & 199 & 495 & 1004 \\
\hline Cauca & 1.1 & * & * & * & 30 & * & 56 \\
\hline Cundinamarca & 8 & 40 & 6.6 & 230 & 200 & 312 & 405 \\
\hline Magdalena & 0.2 & * & * & * & 25 & 13 & 21 \\
\hline Nariño & 0.2 & * & * & * & 5 & * & 18 \\
\hline $\begin{array}{c}\text { Norte de } \\
\text { Santander }\end{array}$ & 90 & 120 & 52.1 & 150 & 123 & 233 & 270 \\
\hline Santander & 10 & 10 & 25.5 & 120 & 105 & 98 & 150 \\
\hline Tolima & 1.0 & (b) & 9.3 & (b) & 60 & 156 & 448 \\
\hline Huila & * & * & * & * & * & * & 51 \\
\hline Valle & 0.6 & 4 & 8.8 & 20 & 50 & 50 & 354 \\
\hline Otros & * & * & 1.6 & * & * & 44 & 36 \\
\hline TOTAL & 114.2 & 230 & 123.8 & 610 & 1085 & 1821 & 3453 \\
\hline
\end{tabular}

Fuente: elaborada por el autor

Se puede observar que durante el último cuarto del siglo XIX la producción de café en Colombia se concentró principalmente en el Norte de Santander. Fue así como en 1874 esta región producía el $79 \%$ del café nacional, debido a que el cultivo había ingresado recientemente importado desde el occidente de Venezuela y logrado imponerse con relativo éxito como actividad de carácter expansiva en el Departamento fronterizo. Para este periodo la producción presentó una fuerte caída debida en gran medida a la Guerra interna de 1876 -1877 y la caída de los precios internacionales, razones por las cuales tan sólo se lograron producir cerca de 52.100 sacos. Las condiciones climáticas, topográficas y físicas favorecieron la expansión del cultivo del café en el Norte de Santander frente a otras opciones agrícolas. En contraste, en Departamentos como
Antioquia y Caldas apenas se lograba producir el $1.05 \%$ del café nacional, puesto que apenas iniciaban la adaptación de su infraestructura agrícola a este nuevo cultivo.

El auge comercial de la frontera derivado en gran medida de la consolidación del comercio de café y la importación de todo tipo de mercancías hacia la región, creó la necesidad de establecer las vías que facilitaran el transporte de grandes cargamentos hacia el Lago de Maracaibo, siguiendo la ruta del puerto venezolano de Encontrados desde donde finalmente se embarcaban las cargas hacia los mercados internacionales.

La importancia del cultivo del café no se debe percibir únicamente al contabilizar el aumento de las exportaciones, sino por la transformación de otros sectores y otras variables claves para el desarrollo 
nacional, tales como el mejoramiento del transporte interno, "su vínculo con el empleo y la situación social, su relación con las finanzas públicas, su impacto en el desarrollo industrial y en la conformación del mercado interno, su incidencia en el desarrollo institucional (...)". (Junguito, 1991, p.25), y sus vínculos con la política nacional.

El aumento del cultivo y comercio del café en el Estado Táchira (Venezuela) y en el Norte de Santander, junto al auge de las importaciones resultantes de esta actividad desde mediados del Siglo XIX, motivaron el mejoramiento de los antiguos caminos de herradura que habían sido utilizados desde tiempos atrás. Se requería adecuar las rutas de tal forma que los hombres y las cargas no sufrieran las consecuencias del largo trecho de viaje entre la región fronteriza y el Puerto de Maracaibo. Adicionalmente el camino debía suplir la carencia de rutas que comunicaran a las poblaciones venezolanas occidentales del Estado Táchira con Maracaibo, ya que para ese momento no se habían adecuado las vías al interior venezolano.

Bajo estas circunstancias ciudadanos venezolanos y colombianos pensaron en la posibilidad de dar inicio a la adecuación de la ruta por tierras nacionales que facilitara la comunicación entre la frontera colombo-venezolana y el Puerto de Maracaibo. La mejor forma que encontraron de cumplir este difícil propósito fue conformando una empresa que gozando de los privilegios otorgados por el Gobierno regional, lograra construir un camino por donde pudieran transitar con más seguridad y rapidez los cargamentos hacia el Puerto. En 1865 fundaron la "Compañía del Camino Carretero á San Buenaventura" que daría inicio a una de las obras más importantes en su tiempo, y sería el inicio de uno de los íconos del transporte regional como lo fue el Ferrocarril de Cúcuta.
De esta manera la sociedad nortesantandereana participó de una revolución que afectó indiscutiblemente la forma de vida, el trabajo, las relaciones comerciales y sociales de San José de Cúcuta y las pequeñas poblaciones cultivadoras de café que posteriormente el Ferrocarril de Cúcuta ensordecería a su paso.

\section{Construcción del camino carretero al puerto de San Buenaventura}

El 23 de Marzo de 1864 don Juan Aranguren, venezolano nacido en Maracaibo, celebró un contrato con el Presidente del Estado Soberano de Santander, José María Villamizar Gallardo para abrir un camino de herradura desde Cúcuta hasta el entonces Puerto de San Buenaventura. La ruta hacia el Puerto de San Buenaventura tenía ciertas ventajas topográficas y económicas sobre otras vías proyectadas durante ese mismo periodo, como la vía del Carare. En primer lugar por la vía cucuteña no había que atravesar ríos importantes, cañones, escarpados o montañas y en segundo lugar casi la totalidad del capital para financiar la construcción de las obras era aportado por los socios privados.

El contrato estipulaba entre otras cosas que el camino debía tener seis metros de ancho, contar con la infraestructura adecuada para el tránsito de mercancías y con los tambos o edificaciones necesarias para alojar en ciertos puntos del trazado, las cargas con destino a la exportación e importación que por allí transitaran. El camino tenía que ser explotado por una compañía radicada en Cúcuta y podía cobrar hasta 90 centavos por el paso de cada carga exportable por un término de 40 años. Por su parte el gobierno nacional le aportaría a la Compañía aparte de los permisos y privilegios respectivos, un total de 40.0000 hectáreas de terrenos baldíos para llevar a cabo el proyecto con todas sus anexidades. 
Hasta ese momento el Ayuntamiento de Cúcuta había cobrado un derecho de peaje y bodegaje por cada carga transportada por el antiguo camino de herradura hacia el puerto aledaño de "Los Cachos" y obtenía de la actividad su principal renta que se acercaba a los 20.000 pesos anuales. El Ayuntamiento de Cúcuta comprendió entonces que la competencia del proyecto de Don Juan Aranguren sería catastrófica para sus intereses y para prevenir la amenaza decidió conformar junto con él la Compañía del Camino de San Buenaventura, el 13 de Mayo de 1865.

Para iniciar la construcción del proyecto el Ayuntamiento aportó el antiguo camino de herradura ya existente al puerto de Los Cachos y las bodegas allí establecidas, el capital de trabajo de la Compañía se fijó en 450.000 pesos que fue dividido en 12 acciones, cada una con un valor de 37.500 pesos, cuatro de las cuales le fueron adjudicadas al Ayuntamiento y una adquirió cada uno de los señores Joaquín Estrada, Felipe Arocha, Idelfonso Urquinaona, Juan Aranguren, el General venezolano Domingo Díaz, Carlos T. Irwin, Andrés Berti Tancredi y Jaime Fossi, casi todos de liberales reconocidos en la región.

El 12 de mayo del mismo año Juan Aranguren le cedió al ex militar venezolano Domingo Díaz, la totalidad de los derechos y obligaciones que le correspondían por el contrato firmado con el Presidente del Estado Soberano de Santander. Al día siguiente de esta transferencia Díaz formó y perfeccionó un nuevo contrato anónimo con el representante del Ayuntamiento de Cúcuta don Manuel Plata Azuero, bajo la denominación de "Compañía Anónima del Camino a San Buenaventura".

Entre las bases más significativas del nuevo contrato estaban que la Compañía asumiera por su cuenta, costo y riesgo la construcción de aquella vía teniendo la preferencia para construir un ferrocarril en el momento que los socios lo estimaran conveniente para los intereses de la Empresa y el comercio de la ciudad. La Compañía quedaba condicionada a entregar la obra terminada en un plazo máximo de 10 años, incluida la construcción de las bodegas necesarias para depositar con seguridad y comodidad un mínimo de veinte mil cargas entre diferentes especies.

El Gobierno del Estado autorizó a la Compañía para que desde la conclusión de la obra cobrara hasta cincuenta centavos de flete por cada bulto no mayor de sesenta y dos kilos y medio que transitara por esta vía con destino a la exportación, y un máximo de ochenta centavos por carga de igual peso que se importara por la vía durante los siguientes cuarenta años; por su parte las cargas de propiedad del Estado podían transitar por el camino sin que la Compañía le cobrara flete alguno.

Paralelamente la Compañía quedó obligada a consignar a partir de la fecha de legalización del contrato, cuatro pesos semestrales de vales de deuda nacional, o el equivalente al doble de esta cantidad en finca raíz hipotecada como garantía del cumplimiento de las obligaciones que contraía. En caso de que la Compañía incumpliera el contrato perdería a favor del Estado el dinero o los bienes que respaldaban el compromiso contraído.

El Gobierno del Estado también le concedió a la Compañía a título gratuito y a perpetuidad catorce mil hectáreas de tierras baldías, de las cuales siete mil pertenecían al Departamento de Cúcuta. También garantizó a los hombres que trabajaran en la construcción del camino, la exención de todos los impuestos públicos directos del Estado o municipales sobre las propiedades que allí establecieran y la excepción del reclutamiento, conscripción o cualquier otro tipo de servicio civil o militar, en tiempos de paz o de guerra por el término de veinte años. 


\section{Conformación de la compañía del ferrocarril de Cúcuta}

Antes de que hubiese finalizado el proyecto de construcción del camino carretero al Puerto, el 16 de marzo de 1875 y días previos al devastador terremoto en Cúcuta (15 de mayo de 1875) se reunió la Junta Directiva del Camino de San Buenaventura en una sesión que fue de trascendental importancia, pues el socio don Arístides García Herreros propuso que el camino construido por la Compañía sirviera de banca para la ruta de un ferrocarril que comunicaría directamente a Cúcuta con el Puerto de San Buenaventura.

De inmediato la idea de construir el ferrocarril fue puesta en consideración de la Compañía y el socio Joaquín Estrada propuso delegar en comisión al Representante del Cabildo en la Empresa, para que presentara un informe habida cuenta de las observaciones que se le habían hecho al proyecto y sobre la conveniencia o no de la obra. Pese a que la mayoría de los socios acogieron la idea con beneplácito y cierto temor inadmisible de ser demostrado ante los demás miembros de la Compañía por tan abrumadora propuesta, también hubo quienes se espantaron con la idea o la percibieron irrealizable en las condiciones propias de la época y de inmediato alegaron su voto en contra de dicho proyecto, tal como sucedió con el Doctor Manuel Plata Azuero.

A medida que el camino hacia el ahora puerto "Villamizar" se convertía en el paso más asequible y obligado de los comerciantes desde y hacia el Lago de Maracaibo en Venezuela, también se veía la necesidad de tomar medidas tendientes a incrementar la importancia de la nueva vía y de paso deshabilitar otras rutas que desde tiempos atrás se habían utilizado para viajar hacia Venezuela y de allí hacia el exterior. En abril de 1868 el mismo doctor Plata Azuero había propuesto pedirle al Presidente del Estado de Santander que le solicitara al Congreso Nacional la habilitación del nuevo Puerto de Villamizar, exclusivamente para el comercio internacional, tan pronto como se hubiera terminado la construcción del camino provisional de herradura.

El 4 de septiembre de 1876 se fundó la Compañía del Ferrocarril de Cúcuta mediante el contrato que se firmó entre Vicente Herrera, Secretario General del Estado Soberano de Santander y José María Villamizar Gallardo ahora representante legal de la Compañía del Ferrocarril de Cúcuta. Mediante la ley 64 del 17 de Junio de 1876 el Congreso Nacional y el Gobierno del Estado Soberano autorizaron a la nueva Compañía del Ferrocarril para convertir en caminos de rieles servido por locomotoras a vapor, la carretera entre San José de Cúcuta y el ahora Puerto Villamizar a orillas del río Zulia.

El gobierno del Estado por medio de contrato concedió inicialmente el privilegio para construir el camino de hierro y explotarlo por un término de noventa y nueve años, contados desde la fecha en que se colocase a servicio público la totalidad de la obra. Pero el contrato inicial de la construcción del Ferrocarril de Cúcuta firmado en septiembre de 1876, fue modificado casi en su totalidad el 12 de enero de 1878, según indica el Archivo Histórico Academia de Historía del Norte de Santander (en adelante AHAHNS) en el año 1929. La Compañía del Camino a San Buenaventura se obligaba a tender en la recientemente construida vía carretera un ferrocarril con un sistema de paralela angosta y de una sola vía de Cúcuta hasta el Puerto de Villamizar, el cual debería cumplir con algunas de las siguientes condiciones: el peso de los rieles debió ser de por lo menos treinta libras por yarda lineal, teniendo estos la forma de 
" $\mathrm{T}$ " y con una distancia de noventa y cinco centímetros (tres pies ingleses) entre las paralelas; el máximo de pendiente no podría superar el cinco por ciento, para lo cual las locomotoras tenían la fuerza suficiente para arrastrar cuarenta y cuatro toneladas de peso bruto, andando a razón de veinte kilómetros por hora en la pendiente máxima (AHAHNS, 1929); también debía adaptar las bodegas que existían para el uso del ferrocarril, construir los edificios y las estaciones secundarias en los lugares de tránsito intermedio; iniciar la operación con, por lo menos, dos locomotoras y treinta carros, incluidos los de pasajeros; construir en Puerto Villamizar un puerto con suficiente solidez para realizar con prontitud la carga y descarga.

El contrato original de construcción del camino carretero firmado en mayo 1865 también contemplaba que si la Compañía decidía acometer la construcción del ferrocarril antes de haber terminado la del camino carretero, se le tenía que adjudicar al Ayuntamiento de Cúcuta el $33 \%$ del capital fijo de la Empresa, equivalente a 150.000 pesos. En el caso de surgir la necesidad de invertir capital adicional en el proyecto, inmediatamente la tercera parte de este monto pasaría a formar parte del capital que el Ayuntamiento tenía en la Compañía.

En la misma medida la Compañía tenía que construir como anexidad del ferrocarril un telégrafo que comunicara las dos extremidades del camino y los lugares intermedios donde hubiera estaciones. En noviembre de 1878 mediante una Ley especial la Asamblea de Estado decretó que las entregas de los trayectos del camino se hicieran en lo sucesivo de la siguiente forma: el 8 de mayo de 1880 dieciocho kilómetros, el 8 de mayo de 1881 otros dieciocho kilómetros y el 8 de mayo de 1882, los dieciocho kilómetros restantes (AHAHNS, 1878). Por medio de esta misma Ley el Gobierno de Estado le cedió a la Compañía a título gratuito treinta y seis mil hectáreas de tierras baldías adicionales, aparte de las cincuenta mil hectáreas de que trataba la Ley nacional No 64 de 1876 y de las catorce mil que pertenecían a la Compañía del camino carretero según el contrato de 15 de junio de 1865; es decir, que el Gobierno nacional le traspasó a la Compañía un total de cien mil hectáreas, tenía la Empresa la atribución de comprobar la calidad de baldíos de los terrenos cedidos, asumiendo tan sólo los gastos necesarios para su adjudicación (AHAHNS, 1929).

Durante el término del privilegio del ferrocarril no se podía gravar sus rentas ni productos (ingresos), ni las obras con alguna contribución directa municipal o del Estado. Así mismo, el Gobierno colocó a disposición de la Compañía del Ferrocarril, según el numeral 6 del Artículo 16, cuando esta solicitara los reos que voluntariamente quisieran ir a trabajar en la obra, era responsabilidad de esta, el suministro de alimentación y asistencia médica.

Se suponía que cumplidos los noventa y nueve años de otorgamiento del privilegio el ferrocarril con todas sus construcciones y anexidades pasarían a ser plena propiedad de la Nación, sin tener la obligación de pagar indemnización alguna a la Compañía que estaba obligada a mantenerlo en buen estado de servicio público. Poco tiempo después de la fundación de la Compañía y de modificarse el contrato de privilegio de construcción del ferrocarril, en 1878 se iniciaron los trabajos de la vía, tropezando con muchas dificultades como la falta de recursos, la insalubridad del clima, los obstáculos inherentes a una obra novedosa en el país, la epidemia de fiebre amarilla que se presentaba en la región, la escasez de mano de obra ingenieril y obrera, y por lo tanto, los elevados costos de los salarios, entre otros factores, de 
acuerdo al Archivo General de la Nación (en adelante AGN) en el año 1892.

El 5 de septiembre de 1878 se colocaron los primeros rieles y tras superar muchos inconvenientes en 1880 se dio al servicio público el primer tramo de la vía férrea que terminaba en la estación de Altoviento en el kilómetro 19, teniendo en servicio tan sólo dos locomotoras y el poco material rodante que hasta ese momento se había importado de Estados Unidos. El segundo tramo de la obra fue el más difícil de construir porque requirió de "fuertes banqueos, cortes y las cuatro más importantes obras de arte" (AGN, 1892, p. 7). Este tramo se abrió al servicio público el 1 de enero de 1884 hasta la estación de Aguablanca en el kilómetro 36.

Desde el 6 de junio hasta el 31 de diciembre de 1885 en el intersticio de guerra civil tras la cual fueron derrotados los liberales, el Gobierno nacional se apropió de la empresa, retardando la construcción del último tramo de la vía y entorpeciendo la totalidad de las labores administrativas. El ejecutivo consideró que las circunstancias de neutralidad requerían que el gobierno tuviera a disposición el mayor número de elementos para devolver la paz a la Nación, fue así como decidió apropiarse de la Compañía férrea arguyendo que era de utilidad pública en esos momentos por la facilidad de transporte que ofrecía el ferrocarril, la Compañía fue inmediatamente asumida y vigilada por los empleados del gobierno de acuerdo con las instrucciones que este transmitió. Para tales fines se hizo uso de la segunda parte del ordinal quince de la Constitución vigente, por el cual se autorizó la expropiación de cosas y entidades como el ferrocarril de Cúcuta (AHAHNS, 1887). Se resolvió expropiar temporalmente la Empresa para ponerla en servicio exclusivo del gobierno nacional y se procedió inmediatamente a recibir el inventario del ferrocarril, sus anexidades y dependencias asegurándose de la administración y dirección la totalidad de la Compañía.

El 19 de diciembre de 1885 siendo Presidente de la República Rafael Núñez se firmó un contrato entre el Secretario de Guerra y Marina Felipe Angulo, autorizado por el Poder Ejecutivo y José A. Céspedes a nombre de la Compañía del Ferrocarril de Cúcuta, mediante el cual el Gobierno nacional le devolvió a la Compañía todos sus bienes, libros, documentos y demás objetos que fueron ocupados por los agentes del Gobierno en virtud de la resolución del Poder Ejecutivo el 27 de mayo del mismo año (AGN, 1885).

En diciembre 17 de 1886 y tras haber pasado veintiún años desde la fundación de la Compañía del Camino a San Buenaventura, el otrora accionista Dámaso Zapata redactó y puso en consideración de la Junta General de Accionistas el proyecto de estatutos para la nueva empresa ferrocarrilera. En este proyecto Zapata, quien en el comienzo había dudado acerca de la idea de construcción del ferrocarril, planteó en primer lugar que se debían derogar los estatutos de la antigua Compañía del Camino aprobados el 13 de diciembre de 1875 y que hasta ese momento habían regido la Empresa, para adoptar los nuevos que empezarían a tener efecto a partir de su inscripción en la oficina de instrumentos públicos del Distrito, dejando atrás la razón social de la Compañía del Camino a San Buenaventura para adoptar el nombre de Compañía del Ferrocarril de Cúcuta.

Finalmente en febrero de 1887 los cucuteños asistieron a la llegada del "el Cúcuta" a la ciudad, así fue bautizado el primer tren que hizo el recorrido completo desde el rio Zulia hasta la ciudad, aunque la inauguración oficial del ferrocarril se hizo el 30 de Junio de 1888. Sin embargo desde el 23 de junio el Presidente de la Compañía le había comunicado con mucho entusiasmo al Señor Presidente 
del Estado de Santander la puesta en servicio público del ferrocarril y que según él traería prosperidad a toda la región fronteriza: "Me complace en participaros, que desde hoy se ha dado al servicio público, el Ferrocarril de Cúcuta es una nueva fuerza que cooperará con la paz a la prosperidad de Santander" (AHAHNS, 1888, p.6).

El costo total de la obra y de sus anexidades al cerrar la cuenta de construcción el 30 de junio de 1888 fue de $\$ 2.132 .341 .91$, cifra que a simple vista parece elevada de acuerdo con el número total de kilómetros construidos en la época, sin embargo, hay que tener en cuenta que en la suma total estaban incluidos los 450.000 pesos del total del capital de la carretera a Puerto Villamizar, los onerosos intereses que adeudaba la Compañía a los pequeños prestamistas que la solventaron en los momentos de difícil consecución de créditos en el extranjero y que arrojó un total de \$741.795.61, cifra que aumentó la cuenta de construcción. Ya en varias ocasiones se había intentado conseguir el crédito en el exterior pero todos los esfuerzos fracasaron y fue tan sólo cuando el éxito de la Compañía parecía ser evidente que se logró conseguir en Bogotá un préstamo, gracias a la gestión de don Francisco Soto Villamizar, liberal ampliamente reconocido (AHAHNS, 1888).

\section{La guerra civil de los mil días y la compañía del ferrocarril}

La última guerra civil colombiana que se presentó a finales del siglo XIX llevó a la Compañía del Ferrocarril a una terrible crisis económica de la cual fue protagonista la Revolución Liberal. EI General Manuel Casabianca fue el militar designado por el Gobierno para dirigir la campaña del Norte y someter a las fuerzas liberales revolucionarias que se formaron en la región, pero tres días después estallar la Guerra, el 20 de octubre de 1899 don Luis Morales Berti, Jefe Civil y Militar de la Provincia de Cúcuta ordenó al Presidente de la Compañía del Ferrocarril don José Agustín Berti no despachar en adelante ningún tren sin previo aviso de la jefatura liberal (Bushnell, 2007, p.18).

Por su parte, el 1 de noviembre de 1899, el revolucionario General Benjamín Herrera también ordenó la suspensión de todo el tráfico de trenes tanto de carga y pasajeros hasta nueva orden por la ruta del ferrocarril de la frontera. Herrera decretó que a partir de esa misma fecha los ingresos o productos del ferrocarril, una vez deducidos los gastos ocasionados por el transporte, debían ingresar a la caja de la intendencia general del Ejército Liberal $^{3}$. Herrera también autorizó la incautación de los rieles y durmientes existentes en los depósitos y talleres de la Compañía y ordenó la entrega de fondos de la Empresa, la ocupación de todas las máquinas, el traspaso de los machetes y todo tipo de herramientas de utilidad en el combate.

José Agustín Berti, Presidente de la Compañía en aquel, momento trató de interceder por los intereses de los socios ante la arremetida liberal y fue puesto en prisión en varias oportunidades por negarse a entregar los fondos que diariamente exigía la Revolución y que estaban destinados al pago de los empleados, los gastos del transporte y el pago de un empréstito contraído en Londres. La toma de la Compañía obligó a sus directivos a someterla a las órdenes de la jefatura rebelde que violó las garantías y exenciones otorgadas por los contratos de privilegio firmados con

3 En las Memorias de la Guerra de los Mil Días Lucas Caballero (2006) apuntó que "las formas de financiar la guerra fueron uno de los secretos mejor guardados, había que defender la identidad de los aportantes y esconder las modalidades delincuenciales con que se armaban los ejércitos" y continuó, "Ios prohombres liberales y fieles seguidores de las banderas revolucionarias sacrifican sus bolsillos y se quitan el pan de la boca". 
el gobierno nacional y departamental, los cuales la eximían de toda contribución e impuesto en tiempos de paz o de guerra.

Ante la arbitrariedad de las medidas impuestas por la jefatura liberal el Presidente de la Empresa José Agustín Berti trató de hacerle entender al jefe militar que la Compañía podía seguir prestándole sus servicios, pero no a cambio del cese de actividades y la expropiación, ya que esto perjudicaba enormemente los intereses de los accionistas, los comerciantes y el público que se movilizaba en el ferrocarril y de paso dañaban el buen crédito que la Empresa tenía con sus acreedores en la región y en el exterior.

Al parecer Herrera no tomó en cuenta las consideraciones hechas por el Presidente de la Empresa y el 2 de noviembre ordenó transportar a Puerto Villamizar todo el café que existía en la Estación Cúcuta, con el propósito de recaudar inmediatamente los fletes de las cargas y poder confiscar estos fondos para cubrir las necesidades de su ejército. No bastando dicha cantidad para suplir los gastos de la guerra en ese momento, Herrera decidió que los comerciantes pagaran anticipadamente los fletes de las 2.734 cargas de café que la Empresa les transportaría al Puerto tan pronto como ellos requirieran el servicio ${ }^{4}$. El 3 de noviembre la Compañía le entregó al Comandante liberal la suma de cinco mil seiscientos ochenta y tres con diecisiete y medio centavos $(\$ 5.683,17 / 2)$ en plata de 0,835 , provenientes de los fletes causados por el café que tenía el comercio de la ciudad con destino a Puerto Villamizar como se puede comprobar en el Archivo Histórico Biblioteca Nacional de Colombia

4 Rudesindo Soto 100 sacos, Bisagno Oliva \& Cía. 20 sacos, Beckman \& Andresen 107 sacos, Breuer Möller \& Cía. 66 sacos, Cruz Sánchez 66 sacos, J. Benöhr \& Cía. 102 sacos, Steinvorth \& Cía. 118 sacos, Pérez y Osorio 446 sacos, Santiago Montaña 903 sacos, Georg Faber 245 sacos, Ricardo Piombino 445 sacos, Van Dissel \& Cía. 128 sacos. (en adelante AHBNC), Sala de Manuscritos Raros, año 1901.

En febrero de 1900 se nombró circunstancialmente a Francisco Soto como Presidente de la Compañía del Ferrocarril y durante los cuatro meses posteriores a su nombramiento, el Ejército Revolucionario del Norte logró obtener de la Empresa gran cantidad de dinero, herramientas y armas que se utilizaron para defenderse de la arremetida gobiernista que se dio en julio de 1900. Durante los cuatro meses de permanencia de Soto en la Presidencia del Ferrocarril, la Jefatura y demás dependencias del Ejército Liberal exigieron a la Compañía la entrega de dinero hasta alcanzar un monto total de $\$ 99.597,65$ en moneda de plata 0,835 , sin tener en cuenta algunos recursos menores que no se llegaron a conocer claramente, materiales y herramientas tales como picas, palas, machetes, crisoles de fundición de metales, alambre y aisladores para el teléfono y el telégrafo, alambre de púas, carbón mineral, hierro colado y todos los durmientes, rieles y las maderas que la Empresa tenía en sus depósitos.

La Revolución utilizó todos los materiales y elementos que "tomó" de los depósitos de la Empresa para fabricar las cerca de diecisiete trincheras, apostadas a lo largo de las calles principales de la ciudad de Cúcuta para tratar de hacerle frente a las tropas oficialistas que en algún momento se tomarían la ciudad. Después del triunfo conservador en Palonegro, el Ejército del Gobierno inició su avance para asediar a los rebeldes liberales que se habían refugiado en Cúcuta. A este asedio se le conoció como el "sitio de Cúcuta" y se inició el 12 de junio de 1900 cuando las tropas del Gobierno ingresaron por el sur a la "Ciudad Amada" y tras cinco semanas de intensos combates, el 16 de julio lograron acabar la toma del Ejército Revolucionario sobre Cúcuta. 


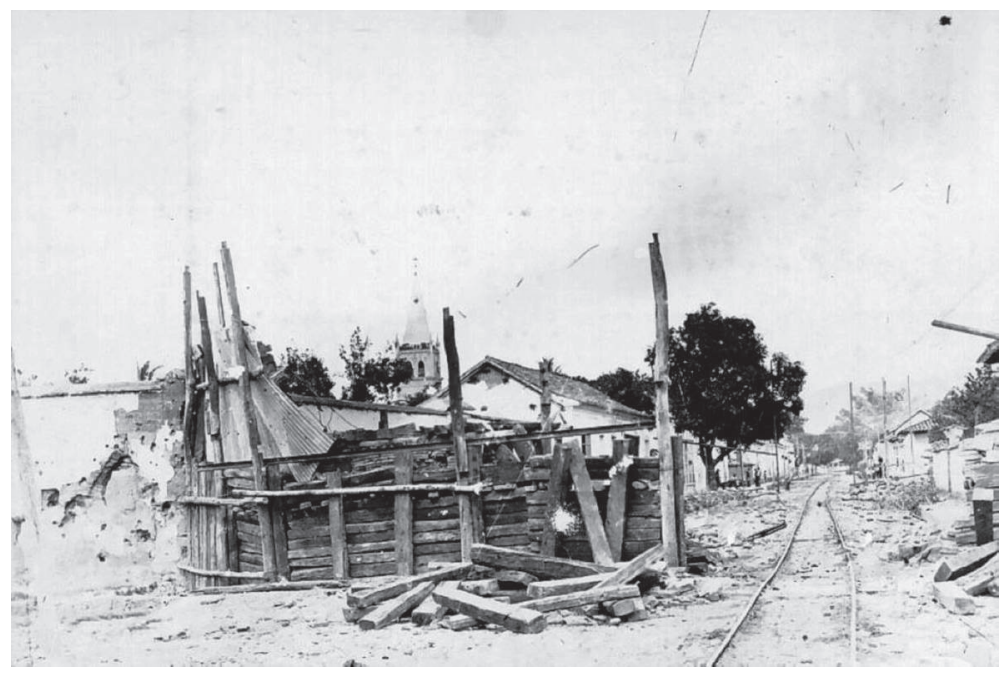

Fuente: Cámara de Comercio de Cúcuta. (2004). Cúcuta a través de la fotografía

Desde el 11 de junio estaban listas las trincheras que encerraban el centro de la ciudad, mientras tanto las familias de filiación liberal eran obligadas a cruzar la frontera hacia Venezuela, pues se presentían los infortunios de la guerra. Efectivamente al día siguiente las tropas oficiales con alrededor de seis mil hombres ingresaron a la ciudad por la vías del Resumen y Los Vados que Ilevaban al puente de San Rafael. Las tropas decidieron atacar por todos los frentes dispuestos de trincheras rebeldes al tiempo que eran apoyados por la artillería que habían instalado en la Piedra de Galembo, al extremo sur de la ciudad.

Tras la derrota de los liberales y por Ilamamiento oficial desde el 16 de julio de 1900 se confió de nuevo la Gerencia de la Compañía al anterior Presidente José Agustín Berti, quien inmediatamente se encargó de presentar el estado de la Empresa basado únicamente en los informes de las Juntas General y Directiva, y en la correspondencia cruzada entre los directivos de la Empresa y los empleados civiles, militares y demás agentes tanto del gobierno como de la revolución. En este momento el movimiento del ferrocarril se limitaba al tráfico local de Puerto Villamizar a Cúcuta y a la frontera con San Antonio del Táchira, razón por la cual los ingresos recibidos por su explotación eran muy bajos y comprometían seriamente el futuro de la Compañía.

\section{Anexidades del ferrocarril de Cúcuta}

La Compañía cucuteña también construyó otros ramales del ferrocarril o anexidades, en abril de 1889 ya se había puesto en servicio el tranvía urbano que buscaba inicialmente facilitar el movimiento de pasajeros y carga dentro de la ciudad y posteriormente comunicar la ciudad con la vecina región venezolana del Estado Táchira mediante el Ferrocarril de la Frontera, tramo que fue terminado en 1897; luego se construyó la ruta hacia el Estado Zulia mediante el empalme del Ferrocarril de Cúcuta con el Gran Ferrocarril del Táchira en Bocas del rio La Grita. En 1921 se quiso acometer la construcción del Ferrocarril del Sur o de Pamplona que pretendió en cierto momento y a 
petición del Gobierno Nacional, unir el Ferrocarril de Cúcuta con los ferrocarriles al interior de la Nación. (Puerto Wilches - Bucaramanga, el Ferrocarril Central del Norte y Tamalameque - Costa Atlántica).

Finalmente el Gobierno Departamental decidió que por la ruta de ferrocarril que conducía a San Antonio del Táchira, era preferible construir una carretera que facilitara la movilización hacia la carretera central del Norte. El servicio decayó hasta que en Noviembre de 1941 el Concejo de Cúcuta ordenó levantar los rieles para iniciar las obras de pavimentación de la vía. Sin embargo, el Ferrocarril de Cúcuta funciono, pese a que el Presidente venezolano Juan Vicente Gómez había puesto en funcionamiento la Gran Carretera de los Andes, que unía a Caracas con San Antonio del Táchira y de paso eliminaba la necesidad de ingresar sus cargamentos al territorio colombiano para transportarlos al lago de Maracaibo. No sobra anotar que para un estudio más detallado sobre el impacto de los proyectos viales venezolanos se recomienda revisar la publicación de la Universidad de los Andes (Mérida) sobre dicha temática, la cual se referencia en la fuentes bibliográficas.

Hacia 1960 el deterioro de las carrileras y máquinas, la disminución del cultivo del café, el aumento del contrabando y la competencia entre el ferrocarril y las carreteras, obligaron a la Compañía a revertir anticipadamente el ferrocarril al gobierno, a desmantelar sus instalaciones y demoler gran parte de la infraestructura de la Compañía.

\section{Conclusión}

La proyección de la construcción del Ferrocarril de Cúcuta en 1876 inició la que tal vez sería la mayor compañía de transporte terrestre de carácter privado que tuvo el país en aquella época y el primer ferrocarril propiamente dicho y tranvía de carácter internacional en Latinoamérica. El auge del cultivo y comercio de café y otras especies en la región fronteriza creó la necesidad de establecer una vía que facilitara el transporte por territorio colombiano hacia el Lago de Maracaibo en Venezuela.

La Empresa del Ferrocarril de Cúcuta a lo largo de su existencia contó con 25 locomotoras, tres líneas férreas, una prolongación y 40 estaciones. Aparte de implementar el Ferrocarril hacia Puerto Villamizar, también llevó a cabo otros proyectos de expansión de la red Férrea en el Departamento y en la frontera Colombo-Venezolana; entre ellos se destacan: el Ferrocarril de la Frontera, el Tranvía urbano, el Ferrocarril del Sur o de Pamplona, la prolongación al rio Grita y el posterior empalme con el Gran Ferrocarril del Táchira.

El Ferrocarril de Cúcuta debe estudiarse como una experiencia distanciada del contexto de los ferrocarriles en Colombia, puesto que tuvo características que lo hicieron único en su tiempo y espacio, como señala David Johnson. “En virtud del potencial comercial, Cúcuta fue declarado Puerto Terrestre, hábil para la importación de mercancías y se estableció allí la Aduana Nacional" (Medina, 1970, p.97).

Se destaca el papel fundamental que cumplieron las casas comerciales que motivaron hasta cierto punto la construcción de caminos y vías férreas en la región, entre ellas se lograron identificar las casas Breüer Moller y Cía., Vandisel Rode y Cía., Bekman y Cía., Caputi y Cía., Rivoli y Cía., Extrada y Cía., Jorge Cristo y Cía., Jorge A. Saiech y Cía., entre otras, y los Bancos de Pamplona, La República, La Mutualidad, Dugan, Mercantil Americano.

En definitiva, es correcto subrayar que la historiografía del transporte, especialmente de los ferrocarriles, no 
muestra continuidad después de 1970; el deterioro y abandono gradual de la red férrea del país durante los últimos veinte años, el advenimiento del transporte aéreo y por carretera no ha generado el interés de los investigadores. Hasta 1920 la función de los ferrocarriles al interior del País había sido integrar la economía al centro y luego al mercado mundial, vinculando las áreas cafeteras con los puertos de embarque, o bien sobre el Magdalena o el mar. Por el contrario la ruta de exportación del café en el Norte de Santander, se había proyectado, en gran medida, al tráfico de cargamentos por los puertos venezolanos, más que por el río Magdalena.

\section{Referencias}

Academia de Historia del Norte de Santander, Archivo Histórico (s.f). Fondo Ferrocarril de Cúcuta. Cúcuta, Colombia.

Academia de Historia del Norte de Santander, Archivo Histórico (s.f). Libro Actas de la Compañía del Camino a San Buenaventura 1865 - 1895. Cúcuta, Colombia.

Academia de Historia del Norte de Santander, Archivo Histórico (s.f). Sesión Ordinaria 7 de Julio de 1868. Cúcuta, Colombia.

Academia de Historia del Norte de Santander, Archivo Histórico (s.f). Sesión Extraordinaria de 8 de Octubre de 1868. Cúcuta, Colombia.

Academia de Historia del Norte de Santander, Archivo Histórico (s.f). Sesión de 29 de junio de 1891. Cúcuta, Colombia.
Academia de Historia del Norte de Santander, Archivo Histórico (s.f). Compañía del Ferrocarril de Cúcuta, Documentos Relativos: Compañía del Camino a San Buenaventura, Ferrocarril a Puerto Villamizar, Ferrocarril a la Frontera y Ferrocarril del Sur. Cúcuta, Agosto 1929. Cúcuta, Colombia.

Academia de Historia del Norte de Santander, Archivo Histórico (s.f). Ley 51 Especial de 4 de Noviembre de 1878, Estado Soberano de Santander. Cúcuta Colombia.

Academia de Historia del Norte de Santander, Archivo Histórico (s.f). Contrato para la devolución de Ferrocarril de Cúcuta, Bogotá, Diciembre 19 de 1885. Cúcuta, Colombia.

Archivo General de la Nación. (AGN). Informe del Presidente de la Compañía al Ministro de Fomento. Tipografía Miguel Lazcano. Cúcuta 1892. Fondo Ferrocarril de Cúcuta. Bogotá, Colombia.

Bushnell, D. (2007). Colombia, una Nación a pesar de sí misma. Bogotá, Colombia: Planeta.

Caballero, L. (2006). Memorias de la Guerra de los Mil Días. Bogotá, Colombia: Alfaguara.

Centro de documentación Biblioteca Nacional de Colombia. (s.f). Informe del Presidente a los Accionistas. Agosto 11 de 1897. Sala 2da No 10306 Pza. 4. Bogotá, Colombia.

Centro de documentación Biblioteca Nacional de Colombia. (s.f). Boletín del Ferrocarril de Cúcuta. Año I. San José de Cúcuta. Abril 22 de 1890. Foto film. Tipografía Miguel Lascano. Bogotá, Colombia.

Junguito, R. (1991). Producción de Café en Colombia. Bogotá, Colombia: Fondo Cultural Cafetero -Fedesarrolllo.

Medina, L. (1970). Cita Histórica Tomo I. Bogotá, Colombia: Anoaqui Impresores.

Solano, G. (1970). 50 Años de vida nortesantandereana. Bogotá, Colombia: Stella.

Universidad de los Andes. (2000) La carretera transandina y sus efectos en la economía venezolana 19101925. Mérida: Autor - Escuela de Economía. 
